\title{
NEW TECHNIQUES IN SPACE RADIO-ASTRONOMY
}

\author{
J. - L. STEINBERG \\ (Paris and Meudon Observatories, France)
}

In spite of the title of this paper, it is not easy to point out many significant developments in the techniques of space radio-astronomy in the last few years. Radio-astronomers are limited in their endeavours by several difficulties which they have not yet been able to overcome completely. I still remember F. G. Smith, in his 1965 Liège Symposium talk, saying that "space radioastronomy is in great danger of turning to geophysics". This statement seems still valid three years later.

However, we might consider that this tendency to deal with geophysical phenomena more than with observations of real astrophysical significance, is after all, not so bad. First of all, it is becoming more and more difficult to define the boundary between geophysics and astrophysics. And second, the Earth environment can be used as a laboratory where a number of processes which are assumed to take place in celestial bodies can be studied in situ. Some types of radio emissions observed by space telescopes are still to be explained and could very well lead to the understanding of mechanisms overlooked by the theoreticians. Radiophysical methods make a very powerful tool to study these phenomena, which occur in a medium that is now being extensively probed by rockets and satellites which send a continuous flow of data to the Earth, about 100000 bits per second, according to Parker.

Also, techniques which were introduced many years ago in geophysics, such as the analysis of radio-propagation phenomena in the ionosphere, are now considered and used for studying other planetary atmospheres and the corona of our Sun. This can be taken as a new branch of space radio-astronomy.

We must not, therefore, be pessimistic about the future of space radio-astronomy; many projects are being planned in many countries and their carrying out will certainly bring important results.

\section{Galactic Radio Emission}

Smith (1965) has shown that, at frequencies close to $1 \mathrm{MHz}$, we are likely to observe emissions of extragalactic origin. This prediction is based on our present knowledge of the spectra of different components of the radio emission observed from the ground at higher frequencies. We know what we should measure, in the absence of any unexpected phenomena. 


\section{A. MEASURING TECHNIQUES AND PROBLEMS}

The difficulties encountered in these measurements of absolute flux densities in space are twofold: (a) Radiation in the Earth's environment or of yet unknown origin, (b) Calibration problems connected with the behaviour of an antenna in a plasma.

(a) 'Abnormal' radiation. - We shall not discuss here radiation observed in the plasma surrounding the space radiotelescope when $1>X>1-Y^{2}$ (where $X$ and $Y$ are the familiar plasma parameters), which is not completely understood but is probably of local origin (Harvey, 1965). But 'abnormal' levels have been observed by Huguenin et al. (1964) at $700 \mathrm{kHz}$, by Benediktov and coworkers (1965) at 725 and $1525 \mathrm{kHz}$, and by Alexander et al. (1966) at $2.7 \mathrm{MHz}$. We have too little information on the characteristics of this radiation to explain its origin. Benediktov et al. have shown it to be correlated with the flux of suprathermal particles of energy higher than $100 \mathrm{eV}$, but the mechanism of production of radio-energy is not completely understood. Huguenin's observations were also taken at fairly high altitudes; they show some bursts occurring sometimes at the same time at 700 and $2200 \mathrm{kHz}$; but the general level observed at $700 \mathrm{kHz}$ remained stable and very high during the whole flight.

Many observations incorporating some directivity and simultaneous measurements on the surrounding medium are necessary to throw some light on this irritating problem.

(b) Antenna behaviour in the plasma. - If we are not making observations at very high altitudes where the operating frequency is very much larger than all resonance frequencies in the surrounding medium, we must investigate the behaviour of our antenna in the plasma.

Extensive theoretical work has been done on the problem in the last few years; the difficulties encountered are formidable, so that a number of simplifying assumptions have to be made, mainly in neglecting the ion sheath which forms on any body immersed in a plasma (Walsh and Haddock, 1964).

The measurement of sky brightness with an antenna in a magnetoplasma has been analyzed by various authors for rather simple cases: uniformly bright sky, plane stratified ionosphere with uniform magnetic field. Two approaches are available: (1) measure the r.m.s. open-circuit noise voltage at the antenna terminal and the antenna impedance: $Z_{\mathrm{a}}=R_{\mathrm{a}}-j / C_{\mathrm{a}} \omega$, then apply Nyquist's theorem (Walsh et al., 1964); or (2) measure the electric field in the medium and the index of refraction of this medium (Budden and Hugill, 1964; Hugill and Smith, 1965). The two methods have been shown to be physically equivalent by various workers (Walsh and Weil, 1967).

However, in the first method, all observers who measured $R_{\mathrm{a}}$ found it much larger than predicted by plasma theory, so that one has to rely on calculations to obtain the real electromagnetic-radiation resistance in the medium from $C_{\mathrm{a}}$ measurements, which lead to the index of refraction. It seems that this method is valid, but will it still be applicable to antennas which can no longer be considered short dipoles and treated 
by quasi-static theory? The only way out is to understand this 'abnormal resistance'. Experiments by Bramley (1965), Walsh et al. (personal communication), Alexander, Stone and Weber (personal communication) and the Meudon Group (1965) have produced data on $Z_{\mathrm{a}}$. The mechanism which produces this 'abnormal resistance' is most probably electron collection in phase with the h.f. potential on the antenna. This is Bramley's conclusion; however, more experiments will have to be carried out before we completely understand the question.

The 'electric-field' method is probably less sensitive than the first one to the presence of this 'abnormal resistance'. But the design of the 'voltmeter' to obtain the open-circuit noise voltage at the antenna terminals must be based on some knowledge of the upper limit of the antenna impedance in the plasma. The above-mentioned experiments show that the measured impedance can be, in some instances, much higher than predicted.

\section{B. RESULTS}

There is little doubt that we shall not be able to 'look' at extragalactic objects on frequencies lower than $1 \mathrm{MHz}$. From all measurements obtained today, it appears that the apparent brightness of the sky decreases with frequency below about $2 \mathrm{MHz}$. However, there is still a large scatter between measurements taken between 1 and 3 $\mathrm{MHz}$, and all we can say of the radio emission in this spectral region is that its spectral index is close to zero. Only one set of measurements has been obtained at frequencies lower than $1 \mathrm{MHz}$, on board Elektron-II, which reached $70000 \mathrm{~km}$ altitude (Benediktov et al., 1965). Only this set of measurements shows, indeed, a fall in the integrated brightness with frequency.

Observations have also been obtained by Slyš (1965) at $30 \mathrm{kHz}, 210 \mathrm{kHz}$ and 1 (or 2) MHz from several Soviet space probes. They show a very steep increase in the brightness with decreasing frequency between 500 and $30 \mathrm{kHz}$. This phenomenon is still unexplained; its existence does not seem doubtful, as it has been measured on several probes carrying different experiments with radio-astronomy receivers; all of these measurements agree to within the experimental uncertainty, ruling out any explanation based on noise from the spacecraft. Similar observations have been taken on board some OGO satellites from 0 to $100 \mathrm{kHz}$ and show also a very high level of noise. But we have no quantitative data on this result.

A tentative explanation based on emission from Jupiter has been put forward by Slyš, but more recent Soviet results do not confirm this hypothesis.

\section{PROSPECTS}

It is clear that, as in early ground-based radio-astronomy, we need some directivity however small; it would give brightness distributions and would help in rejecting and/ or measuring Earth radio radiation, if any. Brightness distribution would in turn 
bring some knowledge of the distribution of ionized hydrogen in the Galaxy which is supposed to produce absorption on low frequencies.

Ionospheric focussing has been proposed by many radio-astronomers as a means to provide directivity (Smith, 1961; Jennison, 1961). It appears that this method is difficult to put into practice as no results have yet been published. The University of Michigan Group has proposed a satellite system based on it, and Ariel-III is also partially designed to use the method. Theories of the phenomenon are available. Most of them do not take into account the Earth's magnetic field or the Earth's curvature. A fortiori, they treat the ionosphere as a continuous well-behaved medium, neglecting inhomogeneities which are known to be present.

The NASA-GSFC Group is planning to launch a series of RAE satellites with a double travelling-wave $\mathrm{V}$ antenna made of four 750-foot booms (Alexander and Stone, 1964). This enormous structure will be gravity-gradient stabilized in a circular orbit at $6000 \mathrm{~km}$ altitude. Each boom is made of thin heat-treated metal which, when driven out of a drum in strip form, takes a tubular section. Recent developments include perforations, silver-plating of the outside surface and blackening of the inside, together with some interlocking device. These characteristics insure very low thermal bending and twisting. Ingenious techniques have been devised to measure current distribution on full scale of these antennas and deduce their polar diagrams in vacuum (Cory and Fenwick, 1966). The RAE booms can be connected in different ways to achieve directivity in the upward vertical direction, the vertical downward direction, and also sideways. This system should give a 20 to $30^{\circ}$ beam at $4 \mathrm{MHz}$ with a frontto-back ratio of better than $15 \mathrm{db}$. It is scheduled for flight in early 1968 .

One can consider that such systems, of still greater size, could be launched to still higher altitudes if gravity-gradient forces are strong enough for their stabilization. Much larger antennas are being studied, mainly in the U.S.A. to our knowledge, which could produce better directivity and front-to-back ratios. They would rely on small satellites, slaved to a master one, all of them with active stabilization to keep the shape of giant rhombic antennas in space and point them (Walsh, personal communication).

If we want more resolving power, the use of aperture synthesis will be necessary. A proposal for such a system has been produced by the Harvard College Observatory Group and by the NASA-GSFC Group (Huguenin, Alexander, personal communications).

\section{CONCLUSION}

The data presently available show that below $4 \mathrm{MHz}$ and down to probably 700 $\mathrm{kHz}$ the sky brightness falls with frequency. This is interpreted in terms of free-free absorption by ionized hydrogen in our own Galaxy.* Below 0.7 MHz, new phenomena

* One is led to an electron measure of $5 \mathrm{pc} \mathrm{cm}^{-6}$. 
occur producing high levels of noise with a density increasing as frequency decreases. No reliable theory is yet available to explain the rise of the apparent sky brightness.

Improvements in the understanding of antennas in a plasma (both for short dipoles and more complicated antennas) are necessary to decrease the spread in low-directivity absolute-brightness measurements below some $\mathrm{MHz}$. The need for some directivity (some tens of degrees or better) is widely recognized, and new systems are readied for flight or being designed to achieve this goal. No systems for better than $10^{\circ}$ beamwidth are being designed to our knowledge, for flight in the next few years.

\section{Solar Studies}

In the past few years, a number of observations of solar radio radiation below 10 $\mathrm{MHz}$ have been made from satellites. Evidence has been obtained that solar bursts of types II and III do, indeed, present spectra extending to frequencies as low as $200 \mathrm{kHz}$.

\section{A. EXPERIMENTS FLOWN}

Most of the published results have been obtained with equipment which was not designed for solar noise observations: Hartz's (1964) pioneering work used the a.g.c. voltage of an ionospheric sounder receiver on board Alouette-I and -II and no onboard calibration or data processing. Slyš (1967) used receivers designed for skybrightness measurements, and the bit rate available to him was very low. Both Hartz and Slyš observations have been related with ground-based spectral records of solar radio emission.

The only experiment flown specifically to study the Sun has been designed by the University of Michigan Group for flight on an EGO satellite. Results have been obtained in the 2 to $4 \mathrm{MHz}$ band with a frequency-swept receiver; they look promising and correlate well with ground-based observations (Warwick, personal communication).

When all these data are completely reduced, they will bring a mine of information on the solar corona at very high altitudes, on the mechanisms which produce solar bursts and the agents which trigger the radiating processes. There is still a lot to be learned about these processes and new phenomena might very well be discovered; for instance, it appears that some bursts show a low-frequency cut-off whose origin is not yet understood.

However, more refined observations than available at present are required; they are being planned.

\section{B. PROJECTS}

We know of at least four projects specifically aimed at solar radio observations at low frequencies. They are: 
Project Pilgrim

Project RAE A and B

Project ATS-E

Project Roseau
Harvard College Observatory (G. R. Huguenin)

NASA-GSFC (R.G. Stone and J.K. Alexander)

NASA-GSFC (R.G. Stone and J.K. Alexander)

R.A. experiment from Meudon Observatory, France

The first three projects are designed and built in the U.S.A. The fourth one is a cooperative project between the U.S.S.R. and France; the satellite will be designed and built in France and launched in the U.S.S.R. in late 1971; it is approved and financed.

The Pilgrim and RAE satellites will carry nothing but radio-astronomy experiments.

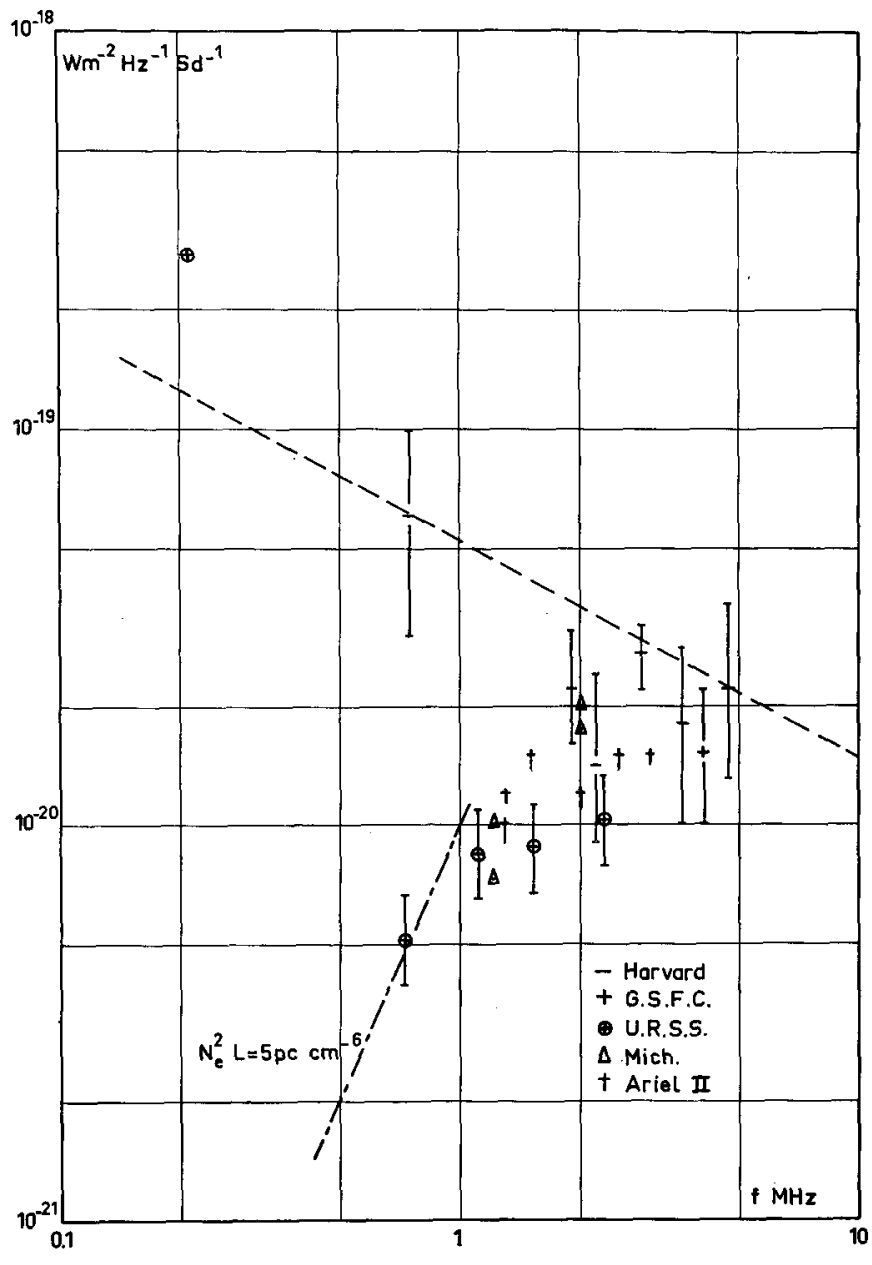

FIG. 1. 

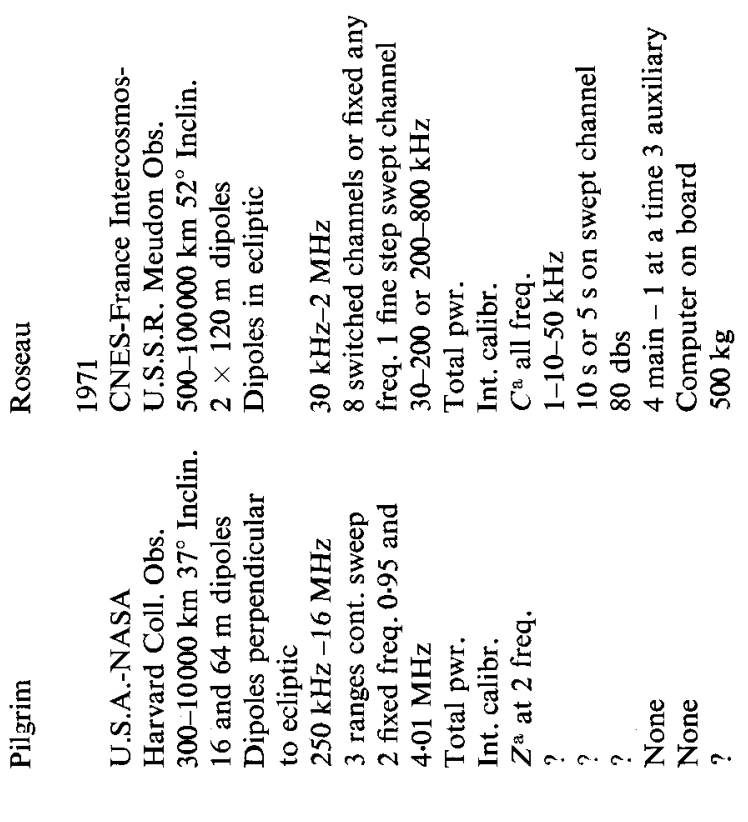

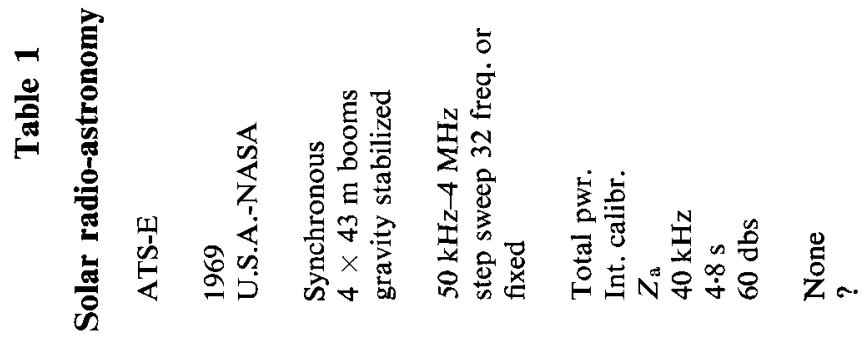

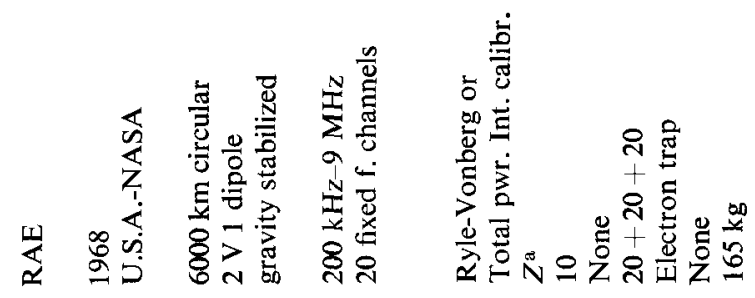

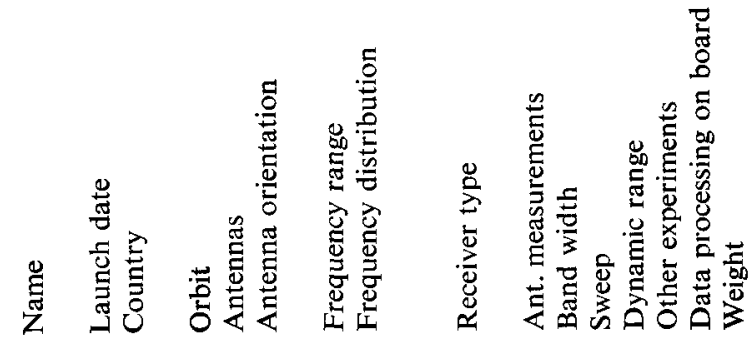


ATS-E will carry other experiments as well (Alexander et al., 1967). Roseau has five experiments on board, only one or two being in use at one time. Table 1 gives a comparison between these four satellite experiments.

A common characteristic is the use of simple dipoles in all projects (we do not consider the RAE V antennas as designed for solar observations). Solar emissions will therefore be modulated by the satellite rotation in both RAE and ATS systems. The Pilgrim project is spin-stabilized around the Sun-Earth axis and will therefore exhibit no such effects. The Roseau satellite, on the contrary, will use spin modulation to get some idea of the direction of solar emissions. Its two crossed dipoles will rotate in the ecliptic plane and can be switched in an attempt to use the dipole directivity to obtain the altitude of radio-emitting regions in the solar corona.

Another common characteristic is the rather poor definition achieved in frequency and time resolution and also in intensity. There is a well-known relationship between bandwidth, time constant (or number of measurements per second), and fluctuations in intensity readings, which is especially annoying in this case where the directivity is poor and the sky background temperature high. On the other hand, near the lower frequency limit of the spectral range covered, the dipole efficiency becomes very small indeed, and therefore the system noise referred to the antenna terminals is no longer negligible.

However, we know so little about solar phenomena at frequencies below $1 \mathrm{MHz}$ that all information, even if rather crude, will be welcome. In the future, better antennas and multichannel receivers will bring more valuable information.

\section{RADIO PROPAGATION EXPERIMENTS}

Observations of the occultations of radio sources by the solar corona have been made for years to study the atmosphere of the Sun. More recently, scintillations of radio sources through the interplanetary medium have been investigated; this kind of work has led to a better knowledge of the inhomogeneities of the interplanetary gas and to measurements of the angular size of radio objects, particularly quasars (Salpeter, 1967; Cohen et al., 1967).

With the advent of satellites, a group in M.I.T. under Professor Harrington, have now proposed to create an artificial radio source to study the solar corona by analyzing the propagation of coherent radio waves through it. This project, named 'Sunblazer', would use a small satellite carrying a modulated transmitter operating on two frequencies, at present 75 and $225 \mathrm{MHz}$. The orbit would be close to the Earth's, inside it, so that the satellite would be 'seen' behind the corona for some time. The idea is to build it with high reliability and low cost in order to be able to launch several of them and always to have one in operation at a given time. By measuring the apparent radio position of this satellite, as well as the polarization and intensity of the radio waves received from it, a great deal of information can be obtained on the corona and the 
interplanetary medium. The effective radiated power from the satellite will, of course, be rather small, so that sophisticated coding on board and receiving techniques on the ground will have to be incorporated in the design.

The experiment will use frequencies which can easily be received on the ground. But it may be necessary to use much smaller frequencies to study, by the same method, the interplanetary medium between the Earth and the limits of the solar system. The receiving end would then have to be on an Earth satellite or on the Moon.

This new technique seems very promising.

\section{Planetary Observations}

All solar low-frequency satellites are, of course, thought to be able to pick up emissions from the planets, if any. The most likely source is Jupiter. However, no emission from this planet has ever been detected from the Alouette satellites, which have provided, by far, the longest time of observation on low frequencies. All surveys of the data have been unsuccessful.

But Alouette equipment was not designed for absolute measurements, so that only bursts of radiation from Jupiter could be detected with any certainty. On the other hand, the Soviet probes Zond-II, Zond-III and Venus-II carried radiometers operating on 30,210 and $2000 \mathrm{kHz}$, with input switching and fed from short dipoles of 3.75 and $2.50 \mathrm{~m}$. Slyš (1966), who was responsible for these experiments, has claimed the observation of Jupiter radiation on $210 \mathrm{kHz}$ on the following grounds:

(1) The $210 \mathrm{kHz}$ radiation varies with the distance between the probe and Jupiter according to an inverse square law. This is based on a comparison between three flights, during which this distance varied widely. This conclusion assumes that Jovian flux density is more or less constant in time.

(2) During the ascent of Zond-II above the Earth's surface, periodic variations of the received signal were noticed, which could be interpreted as due to interference fringes between a direct ray incoming from a discrete source and a ray reflected from the ionosphere. Computation of the zenith distance of the source gives a direction close to Jupiter's at the time of observation.

(3) A drop of $80 \%$ of the received $210 \mathrm{kHz}$ signal at Venus-II has been observed to last for some minutes, after which the received level resumed its previous value. This is interpreted as the occultation of a source by the Moon. Again the source is found to be Jupiter, in spite of the fact that the probe, the Moon, and Jupiter were not lined up at the proper time. This is, rather convincingly, explained in terms of ionospheric refraction and leads to a maximum apparent diameter of Jupiter of $10^{\prime}$.

(4) The low-frequency spectrum seems to fit rather well with higher-frequencies spectra of Jupiter as reported by Ellis.

These observations combined three new techniques in space radio-astronomy:

(1) The use of the inverse-squared distance law to prove the origin of some radia- 
tion from a discrete planetary source. This, of course, can be used only if there is only one such source in the visible sky.

(2) The observation of interference fringes on the ionosphere.

(3) The observation of the Moon occultation of a source of low-frequency radiation.

However, these results can be criticized in many ways. The observation of fringes by reflection on the ionosphere assumes it to be spherically symmetrical and homogeneous. We cannot be sure of that and we even have evidence, at lower altitudes, that it is not the case. The optical path difference at such low frequencies will be so large that it is difficult to believe that coherence between the two interfering rays could be preserved all the way to the satellite. The same applies to the apparent diameter deduced from the Moon-occultation observations. From what we know of the interplanetary medium, we can compute the probable apparent diameter of a point source of $210 \mathrm{kHz}$ radiation as seen through the scattering medium (Salpeter, 1967; Cohen et al., 1967). It is more likely to be some degrees than some minutes. Of course our knowledge of the interplanetary medium between the Earth and Jupiter is rather scanty; however, all we know of the solar wind theory tends to support apparent diameters of the order of degrees with deep intensity scintillations.

In spite of the impressive and ingenious explanations proposed by Slyš, some doubts remain as to whether Jupiter is or is not the source of most of the $210 \mathrm{kHz}$ radiation measured repeatedly on board three different Soviet probes. Moreover, our Soviet colleagues have carried out new experiments which did not confirm Jupiter's radiation at low frequencies.

The Sternberg Institute Group (Shklovsky, personal communication) has flown 3-channel radiometers and short antennas on two lunar orbiters: Luna-XI and -XII. This kind of experiment has been proposed by several groups in different countries but never carried out before. Slyš used the same frequencies as above. In the first orbiter, the antennas were rotating in or near the ecliptic plane. On the second, the spin axis of the satellite was in the ecliptic plane. These two experiments should have provided direct evidence of the presence of a major localized source of radiation, and its position in space should have been measured both by Moon occultation and by spin modulation. Jupiter was not confirmed to be a bright source on $210 \mathrm{kHz}$, and no major source has been found on the frequencies 20-30, 210, 1000-2000 MHz.

All these beautiful Soviet experiments do therefore require reinterpretation, and the task does not seem to be an easy one. Our colleagues must, in any case, be credited for a number of 'firsts' in low-frequency radio-astronomy. Other groups in other countries are already planning new experiments along the same lines, especially lunar occultation experiments.

\section{Conclusion}

A number of new results have been obtained in space radio-astronomy, of which the best established appear to be: the fall in sky brightness below $2 \mathrm{MHz}$, and the 
existence of solar bursts with spectra extending down to $200 \mathrm{kHz}$. None of these results has been obtained with essentially new techniques.

There are indications that interference fringes on the ionosphere and Moon occultation have been observed on $210 \mathrm{kHz}$, but this is still disputable and the results obtained by these methods have not been confirmed.

Lunar orbiters have been used for the first time in low-frequency radio-astronomy. The orbiting of satellites of the Sun to be used as artificial radio-sources of coherent radiation is being planned and deep-space probes are already in use to study the atmosphere of planets (Fjeldbo and Eshelman, 1965).

What we need next is directivity, mainly to investigate the distribution of ionized hydrogen in our Galaxy and get better solar data. We shall certainly see achievements in that direction in the next few years; but it already appears probable that we shall not be able, on frequencies lower than $1 \mathrm{MHz}$, to probe very deep in our Galaxy and that only the immediate neighbourhood of the solar system will be visible to us, in this wavelength range. The question then arises whether it will be better to fly by or to land on the objects we want to study or to put up giant radio-telescopes in space or on the Moon.

\section{Acknowledgment}

I wish to thank the many colleagues who gave me information on their unpublished work and projects.

\section{References*}

Alexander, J. K., Stone, R.G. (1964) Ann. Astrophys., 27, 837.

Alexander, J.K. et al. (1967) Specifications for the solar radioastronomy experiment of the ATS-E Satellite, March.

Alexander, J.K., Weber, R.R., Stone, R.G. (1966) Comm. to 123rd Meeting of the American Astronomical Society.

Benediktov, Ye.A. et al. (1965) Issledovanija Kosmičeskogo Prostranstva, Moscow, June 10-15. - NASA Translation TT F-389.

Bramley, E.N. (1965) Planet. Space Sci., 13, 979.

Budden, K.G., Hugill, J. (1964) Proc. Roy. Soc., A277, 365.

Cory, T.S., Fenwick, R.C. (1966) Comm. to URSI-IEEE. Fall meeting.

Fjeldbo, G., Eshelman, R.V. (1965) J. Geophys. Res., 70, 3217.

Fjeldbo, G., Eshelman, R.V. (1965) J. Geophys. Res., 70, 3701.

Harrington, J.V. (1965) Study of a small solar probe (Sunblazer) PR 5255-5. Center for Space Res., M.I.T.

Hartz, T.R. (1964) Ann. Astrophys. IAU Symp. no. 23, Liège 1964, p. 357.

Harvey, C.C. (1965) Ann. Astrophys., 28, 248.

Hugill, J., Smith, F. G. (1965) Mon. Not. R. astr. Soc., 131, 137.

Huguenin, G. R., Lilley, A.E., McDonough, W.H., Papagiannis, M.P. (1964) Planet. Space Sci., $12,1157$.

Jennison, R.C. (1961) J. Brit. Inst. Rad. Eng., 205.

Meudon Group (1965) Unpublished results on Sept. 1965 radioastronomy rocket flight.

Salpeter, E.E. (1967) Astrophys.J., 147, 433.

* The author does not pretend that this list is complete. 
Slyš, V.I. (1965) Kosm. Issled., 3, 760.

Slyš, V.I. (1966) Kosm. Issled., 4, 924.

Slyš, V.I. (1967) Astron. Zu., 44, 94-97.

Smith, F.G. (1961) Mon. Not. R. astr. Soc., 122, 527.

Smith, F.G. (1965) Mon. Not. R. astr. Soc., 131, 145-153.

Walsh, D., Haddock, F.T. (1964) IAU Symp. no. 23 Liège, p. 335. Astronomical Observations from Space Vehicles, C.N.R.S., Publ. Paris.

Walsh, D., Haddock, F. T., Schulte, H.F. (1964) Space Research, IV. Ed. by P. Muller, NorthHolland Publ. Co., Amsterdam.

Walsh, D., Weil, H. (1967) Radio Science, 2, 225. 